\title{
Reduced Inhibitory Control Mediates the Relationship Between Cortical Thickness in the Right Superior Frontal Gyrus and Body Mass Index
}

\author{
Luca Lavagnino*,', Benson Mwangi', Isabelle E Bauer', Bo Cao', Sudhakar Selvaraj', Alan Prossin' and \\ Jair C Soares' \\ 'UT Center of Excellence on Mood Disorders, Department of Psychiatry and Behavioral Sciences, UT Houston Medical School, Houston, TX, USA
}

\begin{abstract}
Unhealthy eating behaviors often develop in the setting of inadequate inhibitory control, a function broadly ascribed to the prefrontal cortex (PFC). Regulation of inhibitory control by the PFC and its anatomical components and their contribution to increasing body mass index (BMI) are poorly understood. To study the role of PFC in the regulation of inhibitory control and body weight, we examined measures of cortical thickness in PFC sub-regions, inhibitory control (color-word interference task (CWIT)), and BMI in 9I healthy volunteers. We tested the predictive effect of PFC sub-regional cortical thickness on BMI and mediation by inhibitory control measured with CWIT. Measures of depression (BDI-II), anxiety (STAI-T) and trauma-related symptoms (TSC-40) were collected; the disinhibition scale of the three-factor eating questionnaire (TFEQ) was used to assess disinhibited eating. We then tested the relationship between BDII, STAI-T, TSC-40, TFEQ, CWIT, and BMI with correlation analyses. Right superior frontal gyrus cortical thickness significantly predicted BMI $(\beta=-0.91 ; t=-3.2 ; p=0.002)$. Mediation analysis showed a significant indirect effect of cortical thickness on BMI mediated by inhibitory control (95\% Cl=-6.I , -0.67). BMI was unrelated to BDI-II, STAI-T, TSC-40, or TFEQ scores. We found an inverse relationship between cortical thickness in the right-superior frontal gyrus and BMI, which was fully mediated by inhibitory control neurocognitive performance. Our results suggest possible targets for neuromodulation in obesity (ie superior frontal gyrus) and a quantifiable mediator of their effects (ie inhibitory control).

Neuropsychopharmacology (2016) 4I, 2275-2282; doi: I0.I038/npp.2016.26; published online 23 March 2016
\end{abstract}

\section{INTRODUCTION}

Obesity and overweight are major public health concerns (World Health Organization, 2015). Obesity is associated with increased risk of morbidity and mortality from several serious medical illnesses including cancer (Vucenik and Stains, 2012), heart disease ( $\mathrm{Lu}$ et al, 2014), metabolic syndrome (Alberti et al, 2005), and others. Body mass index (BMI) is the most widely used measure to differentiate a healthy body weight $(18.5<\mathrm{BMI}<24.9)$ from overweight $(25<\mathrm{BMI}<29.9)$ and obesity $(\mathrm{BMI} \geqslant 30)$. In obesity, BMI elevation results from an energy imbalance, occurring when calories consumed exceed calories expended. This is often seen in the setting of inadequate self-regulatory control over unhealthy eating behaviors (Appelhans et al, 2011). Given the substantial relationship between unhealthy eating behaviors, elevated BMI, and enhanced risk for serious medical illness, tighter regulatory control over eating

\footnotetext{
*Correspondence: Dr L Lavagnino, Department of Psychiatry and Behavioral Sciences, University of Texas Health Science Center at Houston, 194I East Road, Houston, TX 77054, USA, Tel: + I 281 7|34862624, E-mail: luca.lavagnino@uth.tmc.edu

Received 13 January 20 16; accepted 13 February 20 16; accepted article preview online 18 February 2016
}

behaviors is a critical component to successful treatment of obesity.

An increasing amount of evidence identifies inhibitory control as a critical neurobehavioral correlate of BMI (Vainik et al, 2013). Inhibitory control can be operationalized as overriding a planned or previously initiated action, or as inhibiting the prepotent processing of task irrelevant information (Bari and Robbins, 2013). Some of the standardized neurocognitive tasks available for assessment of inhibitory control include the Stop Signal task, the Go/No Go task and the Stroop task (Chambers et al, 2009). The majority of studies comparing obese and lean subjects found impairments in inhibitory control in the obese group (Nederkoorn et al, 2006; Weller et al, 2008; Davis et al, 2010; Mobbs et al, 2011; Fagundo et al, 2012; Calvo et al, 2014; Mole et al, 2014; Chamberlain et al, 2015; Grant et al, 2015); however, not all studies are in agreement (Nijs et al, 2010; Hendrick et al, 2012; Loeber et al, 2012; Bongers et al, 2014; Hume et al, 2015). Poor inhibitory control performance has been shown to predict future weight gain or lack of success in weight loss during a dietary treatment regimen (Nederkoorn et al, 2007; Pauli-Pott et al, 2010; Weygandt et al, 2013; Kulendran et al, 2014), and is also associated with higher food intake in eating laboratory protocols (Appelhans et al, 2011). 
Binge eating disorder (BED) (American Psychiatric Association, 2013) has a prevalence of $1-3 \%$ in the general population and of about $25 \%$ in obese subjects seeking help for weight loss (Pull, 2004). It is associated with high scores in standardized questionnaires on eating behavior such as the disinhibition scale of the three-factor eating questionnaire (TFEQ) (Hsu et al, 2002) and with substantial psychiatric co-morbidity, most notably anxiety and mood disorders (Hudson et al, 2007). It is currently uncertain whether binge eating is related to impairment in inhibitory control (Davis et al, 2010; Calvo et al, 2014; Maranhão et al, 2015). Enhanced clarity as to the role of binge eating and inhibitory control in weight gain is important in order to facilitate the development of more effective personalized treatment and prevention strategies in obesity.

The prefrontal cortex (PFC) has a critical role in controlling/inhibiting negative impulsive behavior (Szczepanski and Knight, 2014). Lesions within the PFC have been shown to be associated with impaired performance on tasks including the Stroop task (Perret, 1974; Vendrell et al, 1995; Stuss et al, 2001). Among other factors, traumatic experiences have been shown to be associated with reductions in cortical thickness in the PFC (Geuze et al, 2008; Sadeh et al, 2015). The functional role of PFC in regulating food intake is now well established by the findings from functional brain imaging studies in humans. The PFC is recruited when subjects successfully exert self-control in resisting tasty but unhealthy food (Hare et al, 2009), and when subjects use cognitive reappraisal to downregulate their motivation to consume desirable food (Hollmann et al, 2012). Lower baseline prefrontal metabolism has been correlated to higher BMI in both PET (Volkow et al, 2009) and SPECT (Willeumier et al, 2011) studies. Further, greater neural activation to food images in the PFC was found to predict the achievement of lasting weight loss (McCaffery et al, 2009), and less activation in the PFC in another paradigm with food stimuli predicted higher food intake in the following three days (Cornier et al, 2010). Neuromodulation of the PFC with non-invasive brain stimulation techniques (ie transcranial magnetic stimulation, transcranial direct current stimulation) has been proposed as a possible treatment modality in obesity (Alonso-Alonso, 2013; Gluck et al, 2015), therefore investigating which regions of the PFC affect BMI could reveal potential targets for development of novel treatment approaches.

Cortical thickness is a relatively novel brain measure, which is thought to be more specific and biologically relevant than cortical volume, since cortical volume is a composite measure reflecting both cortical thickness and cortical surface area (Winkler et al, 2010). Cortical thickness and cortical surface area are genetically independent features and should probably be analyzed separately whenever possible (Panizzon et al, 2009; Winkler et al, 2010).

Reduction of volumes and cortical thickness have been found in obese subjects in several subdivisions of the frontal cortex: the superior frontal gyrus (Taki et al, 2008; Kurth et al, 2013), middle frontal gyrus (Pannacciulli et al, 2006), inferior frontal gyrus (Taki et al, 2008; Walther et al, 2010; Kurth et al, 2013) and orbitofrontal cortex (Cohen et al, 2011; Maayan et al, 2011; Shott et al, 2014). Importantly, Yokum et al (2012) showed that reduced gray matter volume in the bilateral superior frontal gyrus was related to increased BMI at a 1-year follow-up. Reductions in cortical thickness have also been found in obese subjects in the cingulate cortex, anterior insula, posterior parietal cortex (Hassenstab et al, 2012), in the superior frontal gyrus and orbitofrontal cortex (MarquésIturria et al, 2013), and within occipital, temporal and parietal areas (Veit et al, 2014). However, to date no study has investigated how reduction in cortical thickness affects BMI through neurocognitive function. While Yokum et al (2012) hypothesized that reduced gray matter volume in the prefrontal cortex might increase risk of surrendering to tempting high fat/ sugar foods through a reduction in inhibitory control (Yokum et al, 2012), they did not use neurocognitive measures and therefore could not test this hypothesis.

Identifying a quantifiable mediator (ie inhibitory control) of the impact of PFC alterations on BMI is needed to further enhance translational potential and personalization of novel treatment and prevention strategies across a variety of medical illnesses associated with weight gain.

In the present study, we investigate whether PFC thickness predicts individuals' BMI and whether this relationship is mediated by inhibitory control performance assessed with a neurocognitive task measuring inhibitory control (the 'inhibition' condition of the color-word interference task (CWIT)). Further, we intend to test correlations between a self-report measure of eating disinhibition (the TFEQ 'disinhibition' scale), the CWIT, self-report measures of depression and anxiety (the BDI-II and the STAI-T) and BMI. Based on previous evidence we hypothesized that:

1. cortical thickness will show an inverse correlation with BMI such that reduction in cortical thickness in the PFC will be associated with greater BMI;

2. inhibitory control performance would mediate the relationship between cortical thickness and BMI; and

3. a positive correlation will be found between disinhibited eating (TFEQ), depression and anxiety scores (BDI-II and STAI-T).

\section{MATERIALS AND METHODS}

\section{Sample}

The current study included adults (see Table 1 for demographic information), reducing obvious confounds associated with developmental differences. Only subjects without current axis I diagnosis were included in the study.

The sample was made available by the Nathan-Kline Institute (NKY, NY, USA), as part of the 'original NKIRockland sample'. All approvals regarding human subjects studies were sought following NKI procedures. Scans were acquired from the International Neuroimaging Data Sharing Initiative (INDI) online database (http://fcon_1000.projects. nitrc.org/indi/pro/nki.html).

All subjects were administered the SCID-I-RV/NP (nonpatient edition) to screen for psychiatric disorders (First et al, 2002).

\section{Clinical and Cognitive Measurements}

Available socio-demographics included age, sex, the Wechsler Abbreviated Scale of Intelligence, the Delis-Kaplan Executive Function System including the Color-Word Inference Task or CWIT (Delis et al, 2001), the TFEQ (Stunkard and Messick, 
Table I Demographics and Psychometric Scales

\begin{tabular}{|c|c|}
\hline Measure $(n=91)$ & $N(\%)$ or mean \pm SD (range) \\
\hline Males & $55(60.4 \%)$ \\
\hline Age (years) & $44 \pm 17.9(19-83)$ \\
\hline $\mathrm{BMI}\left(\mathrm{m} / \mathrm{Kg}^{2}\right)$ & $27.2 \pm 5.4(16-40)$ \\
\hline BDI-II & $3.9 \pm 6.3(0-45)$ \\
\hline STAI-T & $30.3 \pm 12.8(0-72)$ \\
\hline TFEQ-disinhibition & $3.6 \pm 2.8(0-12)$ \\
\hline CIWT-inhibition (seconds) & $52.8 \pm 13.3(26-95)$ \\
\hline CIWT-word reading (seconds) & $21 \pm 4.3(12-33)$ \\
\hline CIWT-color naming (seconds) & $27.3 \pm 5.1(16-45)$ \\
\hline TSCL-40 total score & $11.6 \pm \mid 1.4(0-54)$ \\
\hline WASI-full scale intelligence quotient & $\mid 10.1 \pm 12.5(84-138)$ \\
\hline
\end{tabular}

Abbreviations: BDI-II, Beck depression inventory II; BMI, body mass index; CIWT, color word inhibition task; STAI-T, state and trait anxiety inventory-trait anxiety; TFEQ, three factor eating questionnaire; TSCL-40, trauma symptom checklist 40; WASI, Wechsler abbreviated scale of intelligence.

1985), the Beck Depression Inventory-II (BDI-II) (Beck et al, 1996), the State Trait Anxiety Inventory-Trait Anxiety (STAI-T) (Spielberger et al, 1970), the Trauma Symptom Checklist-40 (TSC-40) (Elliott and Briere, 1992). The TFEQ is a self-report tool used to measure dimensions of eating behavior. The second dimension, 'disinhibition', measures disinhibited eating following anxiety, depression or loneliness (Stunkard and Messick, 1985) and it is typically elevated in BED (Hsu et al, 2002).

The CWIT (a modified version of the Stroop task) consists of a series of conditions. In the inhibition condition of the CWIT the subject is asked to name the color the word is written in. However, the colors are printed in an ink that is incongruent with the word (eg, the word 'blue' is printed in red ink). This task condition requires the participant to process task-relevant color information (ink) and inhibit prepotent processing of conflicting task-irrelevant information (word). Performance is measured by completion time: a longer response time reflects worse inhibitory control. In the word reading condition, the subject is asked to read words written in black ink. In the color naming condition, the subject is asked to name the colors of a series of red, green, and blue squares. In our analyses we utilized the reaction time of the inhibition condition as a measure of neurocognitive inhibitory control, and the color naming and word reading reaction times as covariates to control for information processing speed and language/reading ability.

\section{MRI Acquisition Protocol and Pre-Processing}

Three-dimensional magnetization-prepared rapid gradientecho imaging (3D MP-RAGE) structural scans (Mugler and Brookeman, 1990) were acquired using a 3.0 T Siemens Trio scanner with $\mathrm{TR}=2500 \mathrm{~ms}$, $\mathrm{TE}=3.5 \mathrm{~ms}$, Bandwidth $=$ $190 \mathrm{~Hz} / \mathrm{Px}$, field of view $=256 \times 256 \mathrm{~mm}$, flip angle $=8^{\circ}$, voxel size $=1.0 \times 1.0 \times 1.0 \mathrm{~mm}$. More details on image acquisition are available at the website provided above. All T1 scans were visually inspected to ensure the absence of gross artefacts and subsequently pre-processed using the Freesurfer software library (http://surfer.nmr.mgh.harvard.edu/) Version 5.3.0
(Fischl, 2012). The automated segmentation of the prefrontal cortex extracted cortical thickness measures for the following regions: superior frontal cortex, rostral middle-frontal cortex, caudal middle-frontal cortex, pars triangularis, pars opercularis, and pars orbitalis (Desikan et al, 2006).

We averaged the cortical thickness value for the rostral and caudal middle-frontal thickness to obtain a unique measure for the middle-frontal gyrus; we did the same with the pars triangularis, pars opercularis, and pars orbitalis for the inferior frontal gyrus, and with medial and lateral orbitofrontal cortex to obtain a single measurement of the orbitofrontal cortex, reducing the number of variables, while facilitating hypothesis testing.

\section{Data Analysis}

All analyses were conducted with IBM SPSS Statistics (v. 21). Using a linear regression model with cortical thickness measurements of prefrontal regions (superior, middle and inferior frontal gyri, and orbitofrontal cortex), age, and sex as predictors and BMI as outcome, we tested the relationship between cortical thickness and BMI. The regions that were found to predict BMI were then used to test the hypothesis that the relationship between cortical thickness in the PFC and BMI is mediated by inhibitory control performance. Mediation analysis was performed using the PROCESS function in SPSS (Hayes, 2012). This analysis was conducted with bootstrapping, a resampling procedure that constructs confidence intervals for the indirect effect of the mediator. Bias-corrected bootstrap 95\% confidence intervals were obtained with 1000 bootstrapped samples. Subsequent post-hoc analyses included testing associations between the disinhibition scale of the TFEQ and the response time in the inhibition condition of the CWIT (CWIT-inhibition), BMI, BDI-II, and STAI-T.

\section{RESULTS}

Subjects' demographic, psychometric, and neurocognitive data are reported in Table 1. All subjects had at the Wechsler Abbreviated Scale of Intelligence, a Full Scale Intelligence Quotient over 80 (see mean and minimum/maximum values in Table 1). Cortical thickness for the subdivisions of the PFC are reported in Table 2. At the time of data acquisition from the NKI database, T1-weighted MRI scans were available for 141 subjects. Nine subjects were excluded from the analysis because their CWIT inhibition was unavailable. One subject was excluded because of missing weight and height (necessary to calculate BMI). Twenty-three subjects were excluded because younger than 18 years of age. Sixteen subjects were excluded due to presence of Axis I psychiatric diagnosis. One subject was deemed an outlier based on CWIT inhibition score (reaction time was more than six SDs higher than the mean) and excluded from the study. The final sample, on which the analyses were performed, included 91 subjects. All IDs of excluded and included subjects, with reasons for exclusion, are reported in the Supplementary Information.

\section{Multiple Linear Regression Model}

We fitted a regression model using the cortical thickness measurements of the superior, middle and inferior frontal 
Table 2 Cortical Thickness of the PFC in mm

\begin{tabular}{lcr}
\hline Cortical thickness & Left hemisphere mean \pm SD (Range) & Right hemisphere mean \pm SD (Range) \\
\hline Superior frontal gyrus & $2.68 \pm 0.15(2.30-3.02)$ & $2.65 \pm 0.14(2.32-2.97)$ \\
Middle frontal gyrus & $2.44 \pm 0.14(2.05-2.76)$ & $2.41 \pm 0.11(2.10-2.78)$ \\
Inferior frontal gyrus & $2.56 \pm 0.13(2.25-2.93)$ & $2.54 \pm 0.13(2.13-2.89)$ \\
Orbitofrontal & $2.45 \pm 0.11(2.14-2.70)$ & $2.43 \pm 0.13(2.13-2.70)$ \\
\hline
\end{tabular}

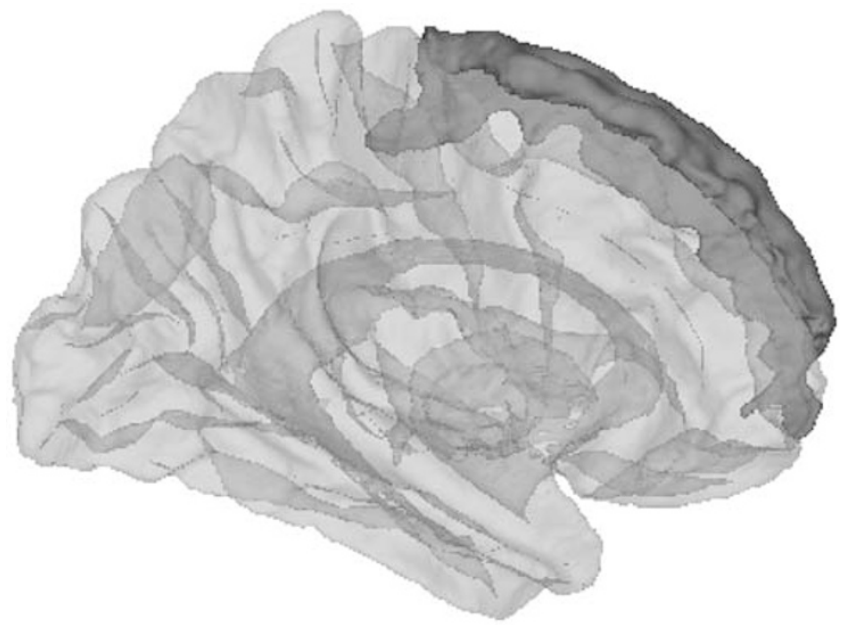

Figure I The right superior frontal gyrus (SFG) in the parcellation obtained with FreeSurfer. The SFG is the only region of the prefrontal cortex (PFC) that is significantly related to the body-mass index (BMI) in the model.

gyrus, orbitofrontal cortex, age, and sex as predictors and BMI as the outcome variable of interest. The omnibus model was statistically significant $\left(F=2.72 ; p=0.006 ; R^{2}=0.25\right)$, with cortical thickness in the right superior frontal gyrus being a significant predictor $(\beta=-0.91 ; t=-3.2 ; p=0.002)$ (Figure 1$)$. Other regions of interest were non-significant $(p>0.05)$.

\section{Mediation of the Effect of Cortical Thickness in the Right Superior Frontal Gyrus on BMI by Inhibitory Control}

We tested our mediation hypothesis with a mediation model, in which inhibitory control (as measured by the CWITinhibition) was postulated to mediate the relationship between cortical thickness in the right superior frontal gyrus (the only significant predictor of BMI in the multiple linear regression model) and BMI (Figure 2). Cortical thickness in the right superior frontal gyrus had an inverse relationship with BMI $(\beta=-9.7, p=0.01,95 \% \mathrm{CI}=-17.1$, -2.2) (Figure 2, Supplementary Figure 1) and an inverse relationship with CWIT-inhibition reaction time $(\beta=-31.5, p=0.0007,95 \% \mathrm{CI}=-49.3,-13.7)$ (Figure 2, Supplementary Figure 2); CWIT-inhibition reaction time had a direct relationship with BMI $(\beta=0.29, p=0.005,95 \%$ $\mathrm{CI}=16.5,25.4$ ) (Figure 2, Supplementary Figure 3 ). The bootstrap analysis of the indirect effect of cortical thickness on BMI showed a bias corrected confidence interval excluding zero (95\% CI $=-6.1,-0.67$ ), demonstrating the presence of an indirect effect of cortical thickness on BMI.

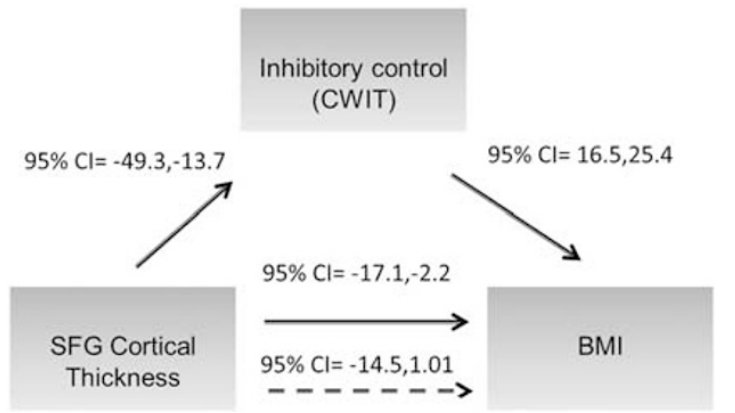

Figure 2 Mediation model and confidence intervals: the relationship between right superior frontal gyrus (SFG) cortical thickness and body-mass index (BMI) (continuous arrow) is no longer significant after controlling for inhibitory control (dashed arrow). Their relationship is therefore fully mediated by inhibitory control.

Notably, the direct effect of cortical thickness on BMI, controlling for CWIT-inhibition reaction time, was no longer significant $(95 \% \mathrm{CI}=-14.5,1.01)$ (Figure 2$)$, indicating that CWIT-inhibition reaction time fully mediates the relationship between cortical thickness in the right superior frontal gyrus and BMI. In order to control for confounding factors, the model was run again using word reading reaction time, color naming reaction time, and the Trauma Symptom Checklist40 Total Score (TSCL-40) as covariates. The model confirmed the presence of an indirect effect $(95 \% \mathrm{CI}=-6.5,-0.6)$, with a non-significant direct effect $(95 \% \mathrm{CI}=-14.7,1.1)$, confirming full mediation when controlling for these factors.

\section{Relationship between the Disinhibition Scale of the TFEQ and BMI, BDI-II and STAI-T}

The correlation matrix between the four variables of interest is reported in Table 3. The TEFQ 'disinhibition of control' eating scale shows a positive correlation with the BDI-II ( $r=0.33, p=0.001)$ and to the total score of the STAI-T $(r=0.26, p=0.013)$. No correlations are shown between the TEFQ disinhibition and the CWIT-inhibition reaction time, with BMI, or with right superior frontal gyrus thickness ( $p>0.05$ for each). Applying a Bonferroni correction for multiple comparisons the correlation between the TFEQ disinhibition of control scale score and the BDI-II remained significant, while the correlation with the STAI-T did not.

\section{DISCUSSION}

We report for the first time that the relationship between cortical thickness of the right superior frontal gyrus and BMI 
Table 3 Correlation Matrix for Neurocognitive and Psychometric Values

\begin{tabular}{|c|c|c|c|c|c|c|}
\hline & CWIT-inhibition & BMI & BDI-II & STAI-T & TFEQ-disinhibition of control & Right SFG cortical thickness \\
\hline CWIT-inhibition & I & $0.29 * * *$ & 0.15 & -0.10 & 0.05 & $-0.35 * *$ \\
\hline $\mathrm{BMI}$ & & 1 & 0.05 & 0.02 & -0.05 & $-0.26 *$ \\
\hline BDI-II & & & । & $0.49 * *$ & $0.32 * *$ & 0.10 \\
\hline STAI-T & & & & I & $0.26 *$ & $0.21 *$ \\
\hline TFEQ- disinhibition of control & & & & & । & 0.04 \\
\hline
\end{tabular}

Abbreviations: BDI, Beck depression inventory; BMI, body mass index; CWIT, color-word interference task; SFG, superior frontal gyrus; STAI-T, state-trait anxiety inventory-trait anxiety.

* $p<0.05$ (two-tailed).

$* * 0<0.01$ (two-tailed).

is completely mediated by CWIT-inhibition reaction time, a measure of inhibitory control, in a population of adult healthy subjects. In other words, inhibitory control entirely accounts for the effect of cortical thickness in the right superior frontal gyrus on BMI (Hayes, 2012). This finding is consistent with the known role of noradrenergic circuits of the PFC in inhibitory control (Szczepanski and Knight, 2014; McKee et al, 2015) and with previous findings of reduction of the cortical thickness in the superior frontal cortex in obesity (Marqués-Iturria et al, 2013). Impairments in Stroop task performance have been shown to be related to reduced dietary adherence (Allan et al, 2011), and to weight gain over a 3-month period (Hotham and Sharma, 2015). Yokum et al (2012) showed that reduced gray matter volumes in the superior frontal gyrus were related to increases in BMI after 1 year of follow-up. The authors hypothesized that gray matter reduction could lead to reduced inhibitory control towards eating foods rich in fat and sugar. The latter may result in a high BMI and ultimately contribute to the development of obesity. Our findings support this hypothesis. Reduced PFC cortical thickness is likely to lead to a failure of the frontal circuits to inhibit orexigenic areas, resulting in failure to dampen hunger and terminate feeding (Le et al, 2007).

We included in our analyses several potential confounding variables (color naming and word reading reaction times; the TSC-40) to control for information processing speed and language/reading ability, and to control for the effects of past trauma, which have been shown to affect the frontal cortex, thus eliminating several potential sources of bias. These analyses point to a specific role of reduced inhibitory control performance in mediating the relationship between reduced cortical thickness and BMI.

Furthermore, in our study CWIT-inhibition was associated with BMI, while the TFEQ eating disinhibition score was not. Conversely, the TFEQ eating disinhibition score correlated to anxiety and depression scores, but not to BMI. These results are consistent with a growing body of evidence that links inhibitory control (assessed with tasks like the Stroop, the Go/No Go and the Stop Signal task) to BMI (Nederkoorn et al, 2006; Mobbs et al, 2011; Fagundo et al, 2012; Calvo et al, 2014; Chamberlain et al, 2015; Grant et al, 2015) and to changes in BMI over time (Nederkoorn et al, 2007; Pauli-Pott et al, 2010; Kulendran et al, 2014). The correlations we identified between TFEQ, BDI, and STAI-T are consistent with the known properties of the TFEQ to measure disinhibited eating behavior occurring in the context of depression and/or anxiety (Stunkard and Messick, 1985). TFEQ-inhibition scale scores have been shown to be higher in extremely obese subjects with BED compared with equally obese controls, suggesting that disinhibited eating behavior is more strongly related to binge eating than to obesity per se (Hsu et al, 2002). The lack of correlations we identified between CWIT-inhibition reaction time and TFEQ eating disinhibition score is in line with findings from studies of group comparisons between equally obese subjects with and without BED, which failed to find between-group differences in inhibitory control performance (Davis et al, 2010; Wu et al, 2013; Mole et al, 2014). Taken together, these findings support the hypothesis that binge eating might be maintained by cognitive factors distinct from those of obesity (Manasse et al, 2015).

Further, we found that inhibitory control performance (assessed with CWIT-inhibition reaction time) was related to BMI but was not associated to depression, anxiety, and disinhibited eating. These findings suggest that it might be necessary to utilize treatments targeted to overweight and the regulation of eating behavior that differ from treatments that are effective for binge eating. A dissociation between eating disinhibition and general inhibitory control would account for the well-replicated clinical finding that successful treatment of binge eating either with selective serotonin reuptake inhibitor (SSRIs) or with cognitive behavior therapy does not usually lead to weight loss (Reas and Grilo, 2008; Capasso et al, 2009; McElroy et al, 2012). In order to obtain improvements in weight, different treatment strategies are needed: facilitating PFC activation might be a promising target. Lisdexamfetamine, a psychostimulant, has been recently approved by the Food and Drug Administration (FDA) for the treatment of BED. Given that psychostimulant drugs are known to increase PFC function and facilitate inhibitory control (Solanto, 1998; Spencer et al, 2001), future longitudinal studies should investigate the effects of this treatment on neurocognitive function and BMI in obese subjects with $\mathrm{BED}$, in addition to its proven efficacy on binge eating (McElroy et al, 2015).

Our study is the first to investigate the relationship between PFC, inhibitory control, and BMI in adults. A previous study in a sample of adolescent subjects found a positive correlation between the TFEQ-inhibition scale and 
the BMI and a negative correlation with the Stroop colorword score and with orbitofrontal (OFC) volume (Maayan et al, 2011). The discrepancy of these findings might be due to several factors. Maayan et al (2011) chose a different region of interest (they focused the analysis on the OFC), and a different measurement (gray matter volume instead of cortical thickness). Moreover, the pattern of late maturation of frontal regions in adolescence (Sowell et al, 1999), and relevant differences in inhibitory-control related activation of the PFC in adolescents compared with adults (Rahdar and Galván, 2014) might be responsible for the discrepancy between Maayan et al's and our results.

Our findings point to opportunities for the development of novel treatment options for obesity and BED. For instance, the right PFC could be a target for non-invasive brain stimulation treatments aimed to improve inhibitory control performance and facilitate dietary self-control (Alonso-Alonso, 2013; Maranhão et al, 2015). If our findings hold true in future replications, the right superior frontal gyrus should be considered a promising candidate for modulatory techniques.

Furthermore, inhibitory control training could be added to treatment programs for obesity. Recent studies provide preliminary evidence that inhibitory control training interventions can reduce food consumption in laboratory settings (Houben, 2011; Delgado-Rico et al, 2012; van Koningsbruggen et al, 2014; Lawrence et al, 2015). Performing general inhibitory control tasks on a regular basis leads to increased activity in the PFC, learning, and rapid plasticity processes (Chambers et al, 2009) that could offset in part the negative effects of low cortical thickness on BMI.

While our findings are novel and robust, it is important to point out some methodological limitations of our study. Although we believe that the current results bear relevance for obesity in BED, it is noteworthy that none of the subjects included in this study had been diagnosed with BED. However, high scores in the TFEQ-inhibition scale have been shown to characterize BED subjects (Hsu et al, 2002). This is consistent with recent studies that suggest the existence of a continuum construct of uncontrolled eating (Vainik et al, 2015). Drawing on these considerations, we believe that the relationships between TFEQ, inhibition scale scores and other variables are likely to be replicated in subjects with BED. Given that our data are cross-sectional, we cannot rule out that reduced cortical thickness might also be a consequence of obesity. However, the study of Yokum et al (2012) showed that reduced gray matter volumes in the superior frontal gyrus and middle frontal gyrus were related to future increases in BMI after a follow-up of one year. For this reason, we believe that our interpretation is plausible. Nevertheless, longitudinal studies are needed to conclusively clarify the causal mechanisms that link cortical thickness, inhibitory control, and weight gain. We analyzed the data using BMI as a continuous dimension because dividing the sample in different BMI groups (eg normal weight, overweight, and obesity) and then performing the analyses would reduce sample size and the power of the analyses. Future studies with a larger sample size should perform separate analyses for different BMI classes (eg normal weight, overweight, and obesity) in order to test whether the relationship between cortical thickness and BMI differs between these groups, or between different degrees of obesity. We did not find an association between BMI and disinhibition that has been shown in some previous studies (Bryant et al, 2008). This could be due to the exclusion of subjects with Axis I diagnoses, resulting in low average disinhibition scores (average $=3.6$, see Table 1 ). It is possible that a sample of subjects with diagnosed Axis I disorders might show an association between disinhibition and BMI; further studies on different patient populations might clarify this issue.

In conclusion, we provided evidence of an inverse relationship between cortical thickness in the right superior frontal gyrus and BMI. This relationship was completely mediated by CWIT, a critical and well-validated measure of inhibitory control performance. Furthermore, in the sample presented, disinhibited eating behavior correlated with depression and anxiety but not BMI.

Taken together, our results provide a framework to characterize behavioral and neurocognitive phenotypes of obesity. The relationship between neuroanatomical (subregional PFC thickness) and neurocognitive variables (CWIT) holds substantial promise for facilitating development of innovative and more effective patient-oriented treatment and prevention strategies in weight-related syndromes.

\section{FUNDING AND DISCLOSURE}

Prof. Soares has participated in research funded by Forest, Merck, BMS, GSK, Johnson \& Johnson and has been a speaker for Pfizer and Abbott. All other authors declare that they have no conflict of interests.

\section{ACKNOWLEDGMENTS}

The present study was supported in part by the Pat Rutherford, Jr. Endowed Chair in Psychiatry (Jair C. Soares).

\section{REFERENCES}

Alberti KGMM, Zimmet P, Shaw J (2005). The metabolic syndrome-a new worldwide definition. Lancet 366: 1059-1062.

Allan JL, Johnston M, Campbell N (2011). Missed by an inch or a mile? Predicting the size of intention-behaviour gap from measures of executive control. Psychol Health 26: 635-650.

Alonso-Alonso M (2013). Translating tDCS into the field of obesity: mechanism-driven approaches. Front Hum Neurosci 7: 512.

American Psychiatric Association. Diagnostic and Statistical Manual of Mental Disorders5th EditionAmerican Psychiatric Publishing; (2013). DSM-5.

Appelhans BM, Woolf K, Pagoto SL, Schneider KL, Whited MC, Liebman R (2011). Inhibiting food reward: delay discounting, food reward sensitivity, and palatable food intake in overweight and obese women. Obesity (Silver Spring) 19: 2175-2182.

Bari A, Robbins TW (2013). Inhibition and impulsivity: behavioral and neural basis of response control. Prog Neurobiol 108: 44-79.

Beck AT, Steer RA, Brown CK (1996). Manual for the Beck Depression Inventory II (BDI-II).

Bongers P, van de Giessen E, Roefs A, Nederkoorn C, Booij J, van den Brink W et al (2014). Being impulsive and obese increases susceptibility to speeded detection of high-calorie foods. Health Psychol 34: 677-685.

Bryant EJ, King NA, Blundell JE (2008). Disinhibition: its effects on appetite and weight regulation. Obes Rev 9: 409-419.

Calvo D, Galioto R, Gunstad J, Spitznagel MB (2014). Uncontrolled eating is associated with reduced executive functioning. Clin Obes 4: $172-179$. 
Capasso A, Petrella C, Milano W (2009). Pharmacological profile of SSRIs and SNRIs in the treatment of eating disorders. Curr Clin Pharmacol 4: 78-83.

Chamberlain SR, Derbyshire KL, Leppink E, Grant JE (2015). Obesity and dissociable forms of impulsivity in young adults. CNS Spectr 20: 500-507.

Chambers CD, Garavan H, Bellgrove MA (2009). Insights into the neural basis of response inhibition from cognitive and clinical neuroscience. Neurosci Biobehav Rev 33: 631-646.

Cohen JI, Yates KF, Duong M, Convit A (2011). Obesity, orbitofrontal structure and function are associated with food choice: a cross-sectional study. BMJ Open 1: e000175.

Cornier M-A, Salzberg AK, Endly DC, Bessesen DH, Tregellas JR (2010). Sex-based differences in the behavioral and neuronal responses to food. Physiol Behav 99: 538-543.

Davis C, Patte K, Curtis C, Reid C (2010). Immediate pleasures and future consequences. A neuropsychological study of binge eating and obesity. Appetite 54: 208-213.

Delgado-Rico E, Río-Valle JS, Albein-Urios N, Caracuel A, González-Jiménez E, Piqueras MJ et al (2012). Effects of a multicomponent behavioral intervention on impulsivity and cognitive deficits in adolescents with excess weight. Behav Pharmacol 23: 609-615.

Delis D, Kaplan E, Kramer J (2001). Delis-Kaplan Executive Function System (DKEFS). San Antonio, Texas.

Desikan RS, Ségonne F, Fischl B, Quinn BT, Dickerson BC, Blacker $\mathrm{D}$ et al (2006). An automated labeling system for subdividing the human cerebral cortex on MRI scans into gyral based regions of interest. Neuroimage 31: 968-980.

Elliott DM, Briere J (1992). Sexual abuse trauma among professional women: validating the trauma symptom checklist-40 (TSC-40). Child Abuse Negl 16: 391-398.

Fagundo AB, de la Torre R, Jiménez-Murcia S, Agüera Z, Granero R, Tárrega $S$ et al (2012). Executive functions profile in extreme eating/weight conditions: from anorexia nervosa to obesity. PLoS One 7: e43382.

First MB, Spitzer RL, Gibbon M, Williams JBW (2002)Biometrics ResearchStructured Clinical Interview for DSM-IV-TR Axis I Disorders, Research Version, Non-patient Edition. (SCID-I/NP). New York State Psychiatric Institute: New York.

Fischl B (2012). FreeSurfer. Neuroimage 62: 774-781.

Geuze E, Westenberg HGM, Heinecke A, de Kloet CS, Goebel R, Vermetten E (2008). Thinner prefrontal cortex in veterans with posttraumatic stress disorder. Neuroimage 41: 675-681.

Gluck ME, Alonso-Alonso M, Piaggi P, Weise CM, Jumpertz-von Schwartzenberg R, Reinhardt M et al (2015). Neuromodulation targeted to the prefrontal cortex induces changes in energy intake and weight loss in obesity. Obesity (Silver Spring) 23: 2149-2156.

Grant JE, Derbyshire K, Leppink E, Chamberlain SR (2015). Obesity and gambling: neurocognitive and clinical associations. Acta Psychiatr Scand 131: 379-386.

Hare TA, Camerer CF, Rangel A (2009). Self-control in decisionmaking involves modulation of the vmPFC valuation system. Science 324: 646-648.

Hassenstab JJ, Sweet LH, Parigi A, Del, McCaffery JM, Haley AP, Demos KE et al (2012). Cortical thickness of the cognitive control network in obesity and successful weight loss maintenance: a preliminary MRI study. Psychiatry Res 202: 77-79.

Hayes AF (2012). PROCESS: A Versatile Computational Tool for Observed Variable Mediation, Moderation and Conditional Process Modeling. at <http://www.afhayes.com/ $>$.

Hendrick OM, Luo X, Zhang S, Li C-SR (2012). Saliency processing and obesity: a preliminary imaging study of the stop signal task. Obesity (Silver Spring) 20: 1796-1802.

Hollmann M, Hellrung L, Pleger B, Schlögl H, Kabisch S, Stumvoll $\mathrm{M}$ et al (2012). Neural correlates of the volitional regulation of the desire for food. Int J Obes (Lond) 36: 648-655.
Hotham S, Sharma D (2015). The relationship between top-down attentional control and changes in weight. Eat Behav 18: 81-83.

Houben K (2011). Overcoming the urge to splurge: influencing eating behavior by manipulating inhibitory control. J Behav Ther Exp Psychiatry 42: 384-388.

Hsu LKG, Mulliken B, McDonagh B, Krupa Das S, Rand W, Fairburn CG et al (2002). Binge eating disorder in extreme obesity. Int J Obes Relat Metab Disord 26: 1398-1403.

Hudson JI, Hiripi E, Pope HG, Kessler RC (2007). The prevalence and correlates of eating disorders in the National Comorbidity Survey Replication. Biol Psychiatry 61: 348-358.

Hume DJ, Howells FM, Rauch HGL, Kroff J, Lambert EV (2015). Electrophysiological indices of visual food cue-reactivity. Differences in obese, overweight and normal weight women. Appetite 85: 126-137.

van Koningsbruggen GM, Veling H, Stroebe W, Aarts H (2014). Comparing two psychological interventions in reducing impulsive processes of eating behaviour: effects on self-selected portion size. $B r$ J Health Psychol 19: 767-782.

Kulendran M, Vlaev I, Sugden C, King D, Ashrafian H, Gately P et al (2014). Neuropsychological assessment as a predictor of weight loss in obese adolescents. Int J Obes (Lond) 38: 507-512.

Kurth F, Levitt JG, Phillips OR, Luders E, Woods RP, Mazziotta JC et al (2013). Relationships between gray matter, body mass index, and waist circumference in healthy adults. Hum Brain Mapp 34: $1737-1746$.

Lawrence NS, Verbruggen F, Morrison S, Adams RC, Chambers CD (2015). Stopping to food can reduce intake. Effects of stimulusspecificity and individual differences in dietary restraint. Appetite 85: 91-103.

Le DSN, Pannacciulli N, Chen K, Salbe AD, Parigi AD, Hill JO et al (2007). Less activation in the left dorsolateral prefrontal cortex in the reanalysis of the response to a meal in obese than in lean women and its association with successful weight loss. Am J Clin Nutr 86: 573-579.

Loeber S, Grosshans M, Korucuoglu O, Vollmert C, Vollstädt-Klein S, Schneider $S$ et al (2012). Impairment of inhibitory control in response to food-associated cues and attentional bias of obese participants and normal-weight controls. Int J Obes (Lond) 36: 1334-1339.

Lu Y, Hajifathalian K, Ezzati M, Woodward M, Rimm EB, Danaei G (2014). Metabolic mediators of the effects of body-mass index, overweight, and obesity on coronary heart disease and stroke: a pooled analysis of 97 prospective cohorts with 1.8 million participants. Lancet 383: 970-983.

Maayan L, Hoogendoorn C, Sweat V, Convit A (2011). Disinhibited eating in obese adolescents is associated with orbitofrontal volume reductions and executive dysfunction. Obesity (Silver Spring) 19: 1382-1387.

Manasse SM, Forman EM, Ruocco AC, Butryn ML, Juarascio AS, Fitzpatrick KK (2015). Do executive functioning deficits underpin binge eating disorder? A comparison of overweight women with and without binge eating pathology. Int J Eat Disord 48: 677-683.

Maranhão MF, Estella NM, Cury MEG, Amigo VL, Picasso CM, Berberian A et al (2015). The effects of repetitive transcranial magnetic stimulation in obese females with binge eating disorder: a protocol for a double-blinded, randomized, shamcontrolled trial. BMC Psychiatry 15: 194.

Marqués-Iturria I, Pueyo R, Garolera $M$, Segura B, Junqué C, García-García I et al (2013). Frontal cortical thinning and subcortical volume reductions in early adulthood obesity. Psychiatry Res 214: 109-115.

McCaffery JM, Haley AP, Sweet LH, Phelan S, Raynor HA, Del Parigi A et al (2009). Differential functional magnetic resonance imaging response to food pictures in successful weight-loss maintainers relative to normal-weight and obese controls. Am J Clin Nutr 90: 928-934. 
McElroy SL, Guerdjikova AI, Mori N, O’Melia AM (2012). Pharmacological management of binge eating disorder: current and emerging treatment options. Ther Clin Risk Manag 8: 219-241.

McElroy SL, Hudson JI, Mitchell JE, Wilfley D, Ferreira-Cornwell MC, Gao J et al (2015). Efficacy and safety of lisdexamfetamine for treatment of adults with moderate to severe binge-eating disorder: a randomized clinical trial. JAMA psychiatry 72: 235-246.

McKee SA, Potenza MN, Kober H, Sofuoglu M, Arnsten AFT, Picciotto MR et al (2015). A translational investigation targeting stress-reactivity and prefrontal cognitive control with guanfacine for smoking cessation. J Psychopharmacol 29: 300-311.

Mobbs O, Iglesias K, Golay A, Van der Linden M (2011). Cognitive deficits in obese persons with and without binge eating disorder. Investigation using a mental flexibility task. Appetite 57: 263-271.

Mole TB, Irvine MA, Worbe Y, Collins P, Mitchell SP, Bolton S et al (2014). Impulsivity in disorders of food and drug misuse. Psychol Med 45: 1-12.

Mugler JP, Brookeman JR (1990). Three-dimensional magnetization-prepared rapid gradient-echo imaging (3D MP RAGE). Magn Reson Med 15: 152-157.

Nederkoorn C, Jansen E, Mulkens S, Jansen A (2007). Impulsivity predicts treatment outcome in obese children. Behav Res Ther 45: 1071-1075.

Nederkoorn C, Smulders FTY, Havermans RC, Roefs A, Jansen A (2006). Impulsivity in obese women. Appetite 47: 253-256.

Nijs IMT, Franken IHA, Muris P (2010). Food-related Stroop interference in obese and normal-weight individuals: behavioral and electrophysiological indices. Eat Behav 11: 258-265.

Panizzon MS, Fennema-Notestine C, Eyler LT, Jernigan TL, PromWormley E, Neale M et al (2009). Distinct genetic influences on cortical surface area and cortical thickness. Cereb Cortex 19: 2728-2735.

Pannacciulli N, Parigi A, Del, Chen K, Le DSNT, Reiman EM, Tataranni PA (2006). Brain abnormalities in human obesity: a voxel-based morphometric study. Neuroimage 31: 1419-1425.

Pauli-Pott U, Albayrak O, Hebebrand J, Pott W (2010). Does inhibitory control capacity in overweight and obese children and adolescents predict success in a weight-reduction program? Eur Child Adolesc Psychiatry 19: 135-141.

Perret E (1974). The left frontal lobe of man and the suppression of habitual responses in verbal categorical behaviour. Neuropsychologia 12: 323-330.

Pull CB (2004). Binge eating disorder. Curr Opin Psychiatry 17: 43-48.

Rahdar A, Galván A (2014). The cognitive and neurobiological effects of daily stress in adolescents. Neuroimage 92: 267-273.

Reas DL, Grilo CM (2008). Review and meta-analysis of pharmacotherapy for binge-eating disorder. Obesity (Silver Spring) 16: 2024-2038.

Sadeh N, Spielberg JM, Logue MW, Wolf EJ, Smith AK, Lusk J et al (2015). SKA2 methylation is associated with decreased prefrontal cortical thickness and greater PTSD severity among traumaexposed veterans. Mol Psychiatry 21: 357-363.

Shott ME, Cornier M-A, Mittal VA, Pryor TL, Orr JM, Brown MS et al (2014). Orbitofrontal cortex volume and brain reward response in obesity. Int J Obes 39: 214-221.

Solanto M V (1998). Neuropsychopharmacological mechanisms of stimulant drug action in attention-deficit hyperactivity disorder: a review and integration. Behav Brain Res 94: 127-152.

Sowell ER, Thompson PM, Holmes CJ, Jernigan TL, Toga AW (1999). In vivo evidence for post-adolescent brain maturation in frontal and striatal regions. Nat Neurosci 2: 859-861.
Spencer T, Biederman J, Wilens T, Faraone S, Prince J, Gerard K et al (2001). Efficacy of a mixed amphetamine salts compound in adults with attention-deficit/hyperactivity disorder. Arch Gen Psychiatry 58: 775-782.

Spielberger CD, Gorsuch RL, Lushene RE (1970). Manual for the State-Trait Anxiety Inventory.

Stunkard AJ, Messick S (1985). The three-factor eating questionnaire to measure dietary restraint, disinhibition and hunger. J Psychosom Res 29: 71-83.

Stuss D, Floden D, Alexander M, Levine B, Katz D (2001). Stroop performance in focal lesion patients: dissociation of processes and frontal lobe lesion location. Neuropsychologia 39: 771-786.

Szczepanski SM, Knight RT (2014). Insights into human behavior from lesions to the prefrontal cortex. Neuron 83: 1002-1018.

Taki Y, Kinomura S, Sato K, Inoue K, Goto R, Okada K et al (2008). Relationship between body mass index and gray matter volume in 1,428 healthy individuals. Obesity (Silver Spring) 16: 119-124.

Vainik U, Dagher A, Dubé L, Fellows LK (2013). Neurobehavioural correlates of body mass index and eating behaviours in adults: a systematic review. Neurosci Biobehav Rev 37: 279-299.

Vainik U, Neseliler S, Konstabel K, Fellows LK, Dagher A (2015). Eating traits questionnaires as a continuum of a single concept. Uncontrolled eating. Appetite 90: 229-239.

Veit R, Kullmann S, Heni M, Machann J, Häring H-U, Fritsche A et al (2014). Reduced cortical thickness associated with visceral fat and BMI. NeuroImage Clin 6: 307-311.

Vendrell P, Junqué C, Pujol J, Jurado MA, Molet J, Grafman J (1995). The role of prefrontal regions in the Stroop task. Neuropsychologia 33: 341-352.

Volkow ND, Wang G-J, Telang F, Fowler JS, Goldstein RZ, Alia-Klein N et al (2009). Inverse association between BMI and prefrontal metabolic activity in healthy adults. Obesity (Silver Spring) 17: 60-65.

Vucenik I, Stains JP (2012). Obesity and cancer risk: evidence, mechanisms, and recommendations. Ann NY Acad Sci 1271: 37-43.

Walther K, Birdsill AC, Glisky EL, Ryan L (2010). Structural brain differences and cognitive functioning related to body mass index in older females. Hum Brain Mapp 31: 1052-1064.

Weller RE, Cook EW, Avsar KB, Cox JE (2008). Obese women show greater delay discounting than healthy-weight women. Appetite 51: 563-569.

Weygandt M, Mai K, Dommes E, Leupelt V, Hackmack K, Kahnt T et al (2013). The role of neural impulse control mechanisms for dietary success in obesity. Neuroimage 83: 669-678.

Willeumier KC, Taylor D V, Amen DG (2011). Elevated BMI is associated with decreased blood flow in the prefrontal cortex using SPECT imaging in healthy adults. Obesity (Silver Spring) 19: 1095-1097.

Winkler AM, Kochunov P, Blangero J, Almasy L, Zilles K, Fox PT et al (2010). Cortical thickness or grey matter volume? The importance of selecting the phenotype for imaging genetics studies. Neuroimage 53: 1135-1146.

World Health Organization (2015). Obesity and overweight. Fact sheet n. 311. Retrieved from http://www.who.int/mediacentre/ factsheets/fs311/en/.

Wu M, Giel KE, Skunde M, Schag K, Rudofsky G, de Zwaan M et al (2013). Inhibitory control and decision making under risk in bulimia nervosa and binge-eating disorder. Int J Eat Disord 46: 721-728.

Yokum S, Ng J, Stice E (2012). Relation of regional gray and white matter volumes to current BMI and future increases in BMI: a prospective MRI study. Int J Obes (Lond) 36: 656-664.

Supplementary Information accompanies the paper on the Neuropsychopharmacology website (http://www.nature.com/npp) 